\title{
Nano-indentation test of electron beam irradiated polyamide 11
}

\author{
Martin Ovsik ${ }^{1, *}$, Lenka Hylova ${ }^{1}$, Ivan Hudec $^{2}$, and Adam Dockal ${ }^{1}$ \\ ${ }^{1}$ Tomas Bata University in Zlin, TGM 5555, 76001 Zlin, Czech Republic \\ ${ }^{2}$ Slovak University of Technology in Bratislava, Institute of Natural and Synthetic Polymers, Vazovova 5, 81243 Bratislava, Slovakia
}

\begin{abstract}
This article deals with the influence of electron beam radiation on nano-mechanical properties and the structure of polyamide 11. Crosslinking of polymers is a process, during which macromolecular chains start to connect to each other and the spatial network creates in the structure. During the action of the ionizing radiation two actions can occur: crosslinking and scission of macromolecules - degradation. Both these processes run parallel. Using the crosslinking technology the standard and construction polymer can obtain the more "expensive" high-tech polymeric materials properties and thus replace these materials in many applications. Tested material was irradiated by different doses of beta radiation (33, 66 and $99 \mathrm{kGy})$. The nano-mechanical properties were measured using DSI method, which fluently records the change of the indentation in time. From this dependence it is possible to determine nano-mechanical properties such as indentation hardness, indentation modulus etc. During results consideration it is obvious that irradiation acts on each polymer differently, but always when the optimal dose was found, nano-mechanical properties increased up to $34 \%$. The changes of nano-mechanical properties were confirmed by structural measurement when the change of hardness and modulus corresponded to gel content.
\end{abstract}

\section{Introduction}

Polyamide 11 (PA 11) is a semi-crystalline thermoplastic polymer belonging to the family of aliphatic polyamides well known as Nylon belonging to the most used polymeric materials. Polyamides are considered as construction polymers used in all branches of the plastics industry. Very often they are used in the automotive industry. Polyamides have excellent mechanical properties, high resistance against chemicals and abrasion etc. These polymers are often reinforced with the fibre glass to obtain better mechanical properties. Other of many possibilities how to improve mechanical properties of polyamides is to use ionizing beta radiation for creation of crosslinked bonds in the inner structure of the material. Radiation crosslinking using ionizing beta radiation is the safe, fast, clean process, free of waste with no environmental hazard. Our team investigated the influence of ionizing beta radiation on the micromechanical properties of PA 11. We found out that after irradiation of PA 11 with the doses of $0,33,66$ and 99 kGy had this polymer the best micro-hardness at the dose of $99 \mathrm{kGy}$, the elastic modulus at the dose of 33 $\mathrm{kGy}$ and the indentation creep at the dose of $99 \mathrm{kGy}$ was similar as in the case of non-irradiated samples [1].

Mária Porubská et al. investigated the influence of gamma irradiation in air and in the inert atmosphere on the unfilled or glass fibre reinforced PA 6. They measured the gel point around the dose of $300 \mathrm{kGy}$ for both materials, but gel formation at higher doses is more effective for pure PA 6. Irradiation in air caused the increase of molecular weight, but the crosslinked portion was not obtained even at highest dose of radiation 500 $\mathrm{kGy}$. The highest increase of impact strength was found out at PA in comparison to PA with glass fibres. The scientist Mária Porubská investigated again PA 6 after irradiation, this time using electron beam radiation. She found out that the crosslinking was more effective for pure PA in comparison with PA filled with the glass fibre [2].

A huge amount of articles is concentrated on the crosslinking of polyamides using various types of crosslinking, but there is a small amount of articles concentrated on crosslinking of PA 11 using electron beam radiation and subsequent investigation how micromechanical properties were changed after this irradiation $[3,4]$.

Common PA11, when exposed to the effect of the radiation crosslinking, degrades and its mechanical properties deteriorate. Using crosslinking agent TAIC (triallyl isocyanurate) produces a crosslinking reaction inside the PA11 structure. The utility properties of PA11 improve when the non-crystalline part of PA11 is crosslinked (Figure 1).

The thermoplastics which are used for production of various types of products have very different properties. Standard polymers which are easy obtainable with favorable price conditions belong to the main class. The disadvantage of standard polymers is limited both by mechanical and thermal properties. The group of standard polymers is the most considerable one and its share in the production of polymers is as high as $90 \%$. 


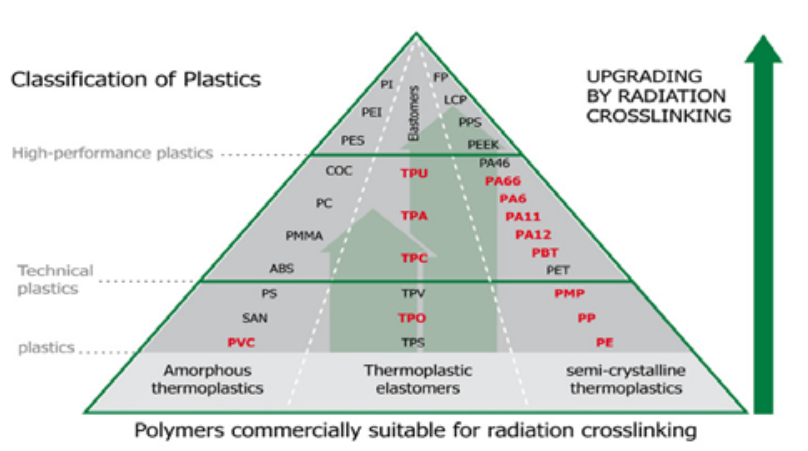

Fig. 1. Upgrading properties by radiation crosslinking

The main aim of this study is to compare changes in the nano-mechanical properties of irradiated polyamide 11. This work explores the potential improvements in nano-indentation properties and morphology of crosslinked polyamide 11 by ion beam treatment. These tested polymers find a broad application in many branches of industry and in daily life as well.

\section{Experimental}

The crosslinked material was chosen for the measurement of nano-indentation properties for comparison of various options. Polyamide 11 from the company PTS was chosen as a material. Testing samples were made from this material according to a standard ISO 527-2 1BA for the tensile test. The processes of injection molding and radiation crosslinking were performed with the minimum time gap to avoid the influence on the measurement surroundings.

\subsection{Material}

For this experiment polyamide 11 V-PTSCREAMID11T*M600/13 (PTS Plastics Technologie Service, Germany) was used. The material already contained the special crosslinking agent TAIC - triallyl isocyanurate (6 volume \%), which should enable subsequent crosslinking by ionizing $\beta$-radiation.

\subsection{Sample preparation}

Process conditions were set according to the manufacturer. These samples were moulded by the injection moulding machine ARBURG Allrouder $470 \mathrm{H}$ (Loßburg, Germany) according to the same process conditions. The normalized specimens, with dimensions of $(80 \times 10 \times 4) \mathrm{mm}$, were used. Process conditions; see Table 1.

Table 1. Process parameters.

\begin{tabular}{ccc}
\hline Parameters & Unit & PA 11 \\
\hline Injection Pressure & $\mathrm{MPa}$ & 65 \\
Cooling Time & $\mathrm{s}$ & 17 \\
Mould Temperature & ${ }^{\circ} \mathrm{C}$ & 60
\end{tabular}

\begin{tabular}{ccc} 
Zone 1 & ${ }^{\circ} \mathrm{C}$ & 240 \\
Zone 2 & ${ }^{\circ} \mathrm{C}$ & 250 \\
Zone 3 & ${ }^{\circ} \mathrm{C}$ & 260 \\
Zone 4 & ${ }^{\circ} \mathrm{C}$ & 270 \\
\hline
\end{tabular}

\subsection{Irradiation process}

The crosslinking causes the connection of polymeric chains to each other, most often using covalent bonds to form the spatial network. Test bodies were irradiated under industrial conditions on a commercially available irradiation device in a broader range of radiation doses $(0,33,66$ and $99 \mathrm{kGy})$ compared to the doses corresponding to the experience in the practice.

All samples were irradiated with electron (beta) rays (accelerated electrons - A Rhodotron R E-beam accelerator, electron energy $10 \mathrm{MeV}$ ) in the firm BGS Beta Gamma Service GmbH \& Co, Saal am Danau Germany.

\section{$1.4 \mathrm{Gel}$ content}

A gel test is conducted to determine the content of nonfiltered phase-gel of the given material according to the CSN EN 579 standard. The portion of $1 \mathrm{~g}$ (of material radiated by radiation doses), weighed with a precision of three decimal places, was mixed with $100-250 \mathrm{~mL}$ of solvent. Xylol was used for testing the polymers because it dissolves the amorphous part of polymers; the crosslinking part does not dissolve. The mixture was extracted for $6 \mathrm{~h}$. Then, the solutes were separated by distillation. After removing the residual xylol, the crosslinked extract was rinsed in distilled water. The rinsed extract was then dried for $8 \mathrm{~h}$, under vacuum, at $100{ }^{\circ} \mathrm{C}$. The dried and cooled residue was re-weighed, with a precision of three decimal places, and compared to the original weight of the portion. The result, stated in percentage, shows the degree of crosslinking:

$$
G_{i}=\frac{m_{3}-m_{1}}{m_{2}-m_{1}} \cdot 100
$$

Where $\mathrm{G}_{\mathrm{i}}$ is the degree of crosslinking of each specimen expressed in percentage, $\mathrm{m}_{1}$ is the weight of the cag and lid in milligrams, $\mathrm{m}_{2}$ is the total of weights of the original specimen, cage and lid in milligrams, and $\mathrm{m}_{3}$ is the total of the weights of the residue of specimen, cage and lid in milligrams.

\subsection{Nano-indentation test}

Nano-indentation properties was measured by means of Nano-Hardness Tester $\mathrm{NHT}^{3}$ (Fig. 2) made by Anton Paar (Graz, Austria), according to the CSN EN ISO 14577 standard. On each material at least ten indents were made and the results were statistically treated. Standard simple loading-unloading mode was used. From the obtained "load vs. depth of penetration" curves values of nano-indentation properties were calculated 
according to Oliver and Pharr. The measurement parameters are shown in Table 2.

Table 2. Measurement parameters.

\begin{tabular}{ccc}
\hline Parameters & Unit & Value \\
\hline Maximum Load & $\mathrm{mN}$ & 10 \\
Load/Unload Speed & $\mathrm{mN} / \mathrm{min}$ & 20 \\
Holding Time & $\mathrm{s}$ & 90 \\
\hline
\end{tabular}

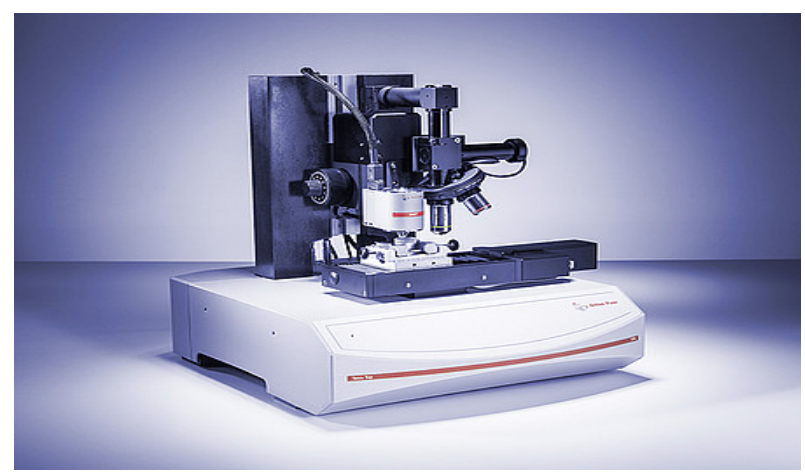

Fig. 2. Nano-indentation Tester $\mathrm{NHT}^{3}$.

The indentation hardness $\left(\mathrm{H}_{\mathrm{IT}}\right)$ was calculated as maximum load $\left(\mathrm{F}_{\max }\right)$ to the projected area of the hardness impression $\left(\mathrm{A}_{\mathrm{p}}\right)$ and the indentation modulus $\left(\mathrm{E}_{\mathrm{IT}}\right)$ is calculated from the Plane Strain modulus $\left(\mathrm{E}^{*}\right)$ using an estimated sample Poisson's ratio ( $v$ ) according to $[5-8]$ :

$$
\begin{gathered}
H_{I T}=\frac{F_{\max }}{A_{p}} \\
E_{I T}=E^{*} \cdot\left(1-v_{s}^{2}\right)
\end{gathered}
$$

\section{Results and discussion}

For the nano-mechanical properties measurement the polyamide 11 was selected for radiation crosslinking. The main reason of this selection is this easy modification by beta radiation and this often application in the technical practice. For confirmation of the results and changes of nano-mechanical properties of tested polymers using DSI method, structural measurements were done. These properties were always 10 times measured at polymers. The influence of radiation dose on nano-mechanical properties of tested polymers was compared.

The measured values of the ultra-nano indentation test were obtained for PA 11 irradiated with beta rays as shown in Table 3.

Table 3. Nano-indentation values

\begin{tabular}{cccccc}
\hline Parameters & Unit & 0 kGy & 33kGy & 66kGy & 99kGy \\
\hline Gel Con. & $\%$ & 0,00 & 33,00 & 87,00 & 76,00 \\
$\mathrm{H}_{\mathrm{IT}}$ & $\mathrm{MPa}$ & 153,38 & 156,08 & 205,19 & 176,46 \\
$\mathrm{E}_{\mathrm{IT}}$ & $\mathrm{GPa}$ & 2,11 & 2,16 & 2,50 & 2,18 \\
\hline
\end{tabular}

Nano-indentation characteristics determined by DSI method are depicted in Figure 3. From Figure 3, there is evident the change of the properties of polyamide 11 with addition of crosslinking agent before and after irradiation by different doses. Figure characterizes the dependence of the indentation depth on time of the application of the constant loading force, while indentation curves give the possibility to obtain indentation hardness, indentation modulus and the size of deformation, elastic, plastic and total work needed to formation of the indentation.

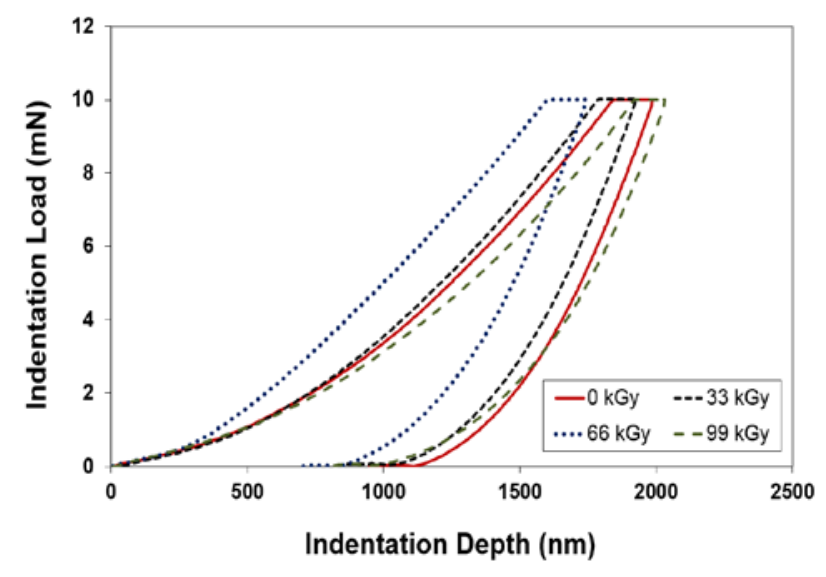

Fig. 3. Indentation load vs. Indentation depth

Graphic evaluation of measured results of indentation hardness in the dependence on different radiation doses for polyamide 11 are shown in Figure 4. From measured values of indentation hardness follows that radiation crosslinking at polyamide 11 takes effect by higher hardness at tested polymers. The lowest value of indentation hardness was found at non-irradiated PA $11(153.4 \mathrm{MPa})$. The highest value of indentation hardness was measured at PA 11 irradiated at the dose of $66 \mathrm{kGy}$. The difference in hardness at tested PA 11 (205.2 MPa) was approximately $34 \%$. According Figure 4 it is clear that indentation hardness at tested polymers is strongly influenced by beta radiation. The values drop of hardness after application of radiation doses higher than $66 \mathrm{kGy}$ is probably caused by material degradation after irradiation.

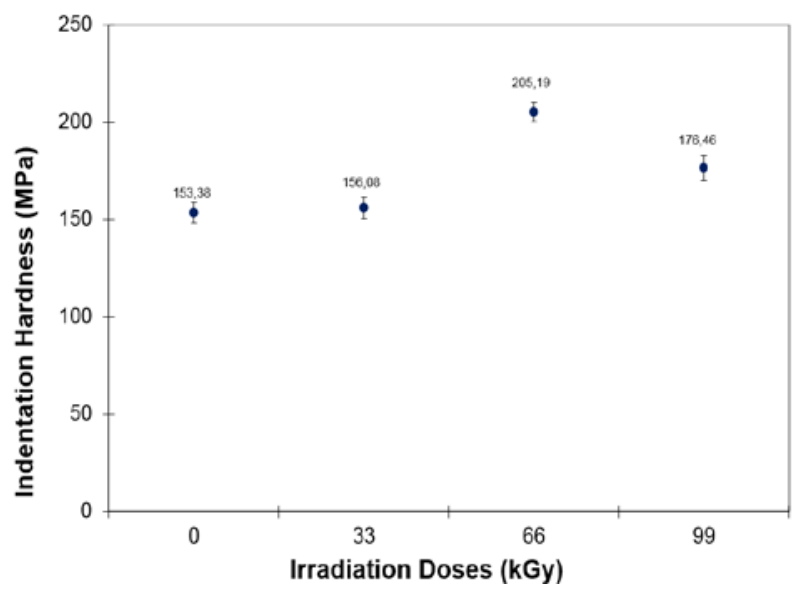

Fig. 4. Indentation hardness vs. Irradiation doses 
PA 11 indentation modulus increased with the radiation dose up to maximum at the dose of $66 \mathrm{kGy}(2.5$ $\mathrm{GPa}$ ) what is approximately higher by $19 \%$ in comparison with non-irradiated PA 11 (2.1 GPa). The higher radiation dose, the lower values of indentation modulus (Figure 5).

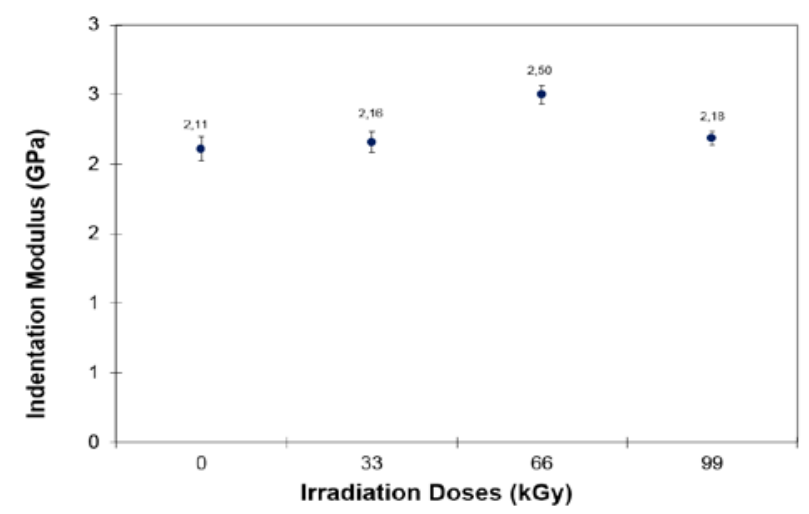

Fig. 5. Indentation modulus vs. Irradiation doses

The gel test is carried out for the purpose of measurement of non-filterable phase content - the gel of given material according to the standard EN ISO 579. The determination of the gel content at Polyamide 11 in the dependence on applied dose of radiation is obvious from the Figure 6.

Figure 6 shows the resultant content of PA 11 gel. The trend in this material is similar to nano-mechanical properties trend, there is also a significant increase in the gel content at a radiation dose of $66 \mathrm{kGy}(87 \%)$ and with an increasing radiation dose the gel content is slightly decreased up to a maximum radiation dose of $99 \mathrm{kGy}$ where the gel content was measured at $76 \%$. These results correspond to the measurement of nanomechanical properties, where the highest values were measured at the radiation dose of $66 \mathrm{kGy}$.

The accuracy of the gel content measurement is largely dependent on the robustness of the filter screen used, and so the resulting "micro gels" of the material in the lower radiation dose can pass through the filter sieve, so they are not counted in the total gel content. However, they already play a significant role in the measured properties.

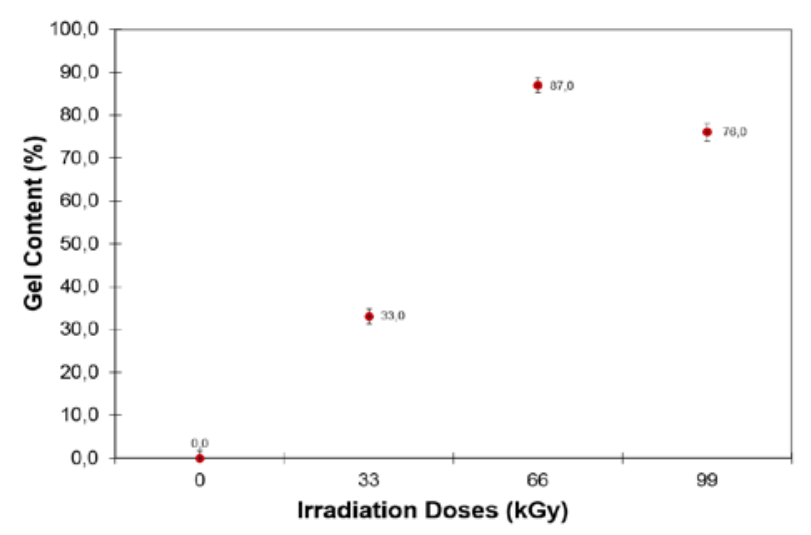

Fig. 6. Gel content vs. Irradiation doses

\section{Conclusions}

From the results it is evident that beta radiation crosslinking has a influence on nano-mechanical properties of polymers (PA 11). Nano-mechanical properties (indentation hardness and indentation modulus) were measured using DSI method at all tested polymers. Polyamide 11 irradiated at the dose of $66 \mathrm{kGy}$ reached the biggest changes of tested properties in comparison with non-irradiated materials. Nevertheless, it is necessary to mention that the final dose of radiation is always necessary to consider thoroughly with respect to the efficiency of the irradiation process. In some cases it is possible to choose also the lower radiation dose with comparable reached results of modification. Thanks to different applied radiation doses the improvement of usual material properties occurs, thereby the cheaper commodity or construction material can replace more expensive construction or special polymers.

The results of nano-mechanical properties were supported by the measurement of the structure, where measurements of gel test were performed. The Gel content confirmed the influence of radiation doses on the structure of studied polymers. From the measurements it can be stated that the highest changes were achieved by polyamide 11 examined at higher doses of radiation.

It should also be noted that a higher dose of radiation intensity does not necessarily mean a higher improvement in desired properties. For a particular application and material, it is always necessary to look for a suitable dose of radiation intensity.

This work was supported by the European Regional Development Fund under the project CEBIA-Tech Instrumentation No. CZ.1.05/2.1.00/19.0376 and by the Ministry of Education, Youth and Sports of the Czech Republic within the National Sustainability Program project no. LO1303 (MSMT-7778/2014). Moreover, it was supported by the Internal Grant Agency of TBU in Zlin: no. IGA/FT/2018/012.

\section{References}

1. M. Ovsik, M. Stanek, M. Reznicek, MATEC Web of Conferences 125, (2017).

2. M. Porubská, D. Babić, I. Janigová, M. Šlouf, K. Jomová, I. Chodák, Polym. Bull 73, (2016).

3. M. Porubská, O. Szöllös, I. Janigová, K. Jomová, I. Chodák, Radiat. Phys. Chem. 133, (2017).

4. T. Yu, J. Chen, F. Wu, J. Rocks, Mater. Sci. Forum 815, (2015).

5. Drobny J.G., Radiation Technology for Polymers (CRC Press, New York, 2003).

6. G. Zamfirova, V. Gaydarov, T. Zaharescu, L. G. Silva, Chemicke Listy 104 (2010).

7. T. Metanawin, A. Jamjumrus, S. Metanawin, Morphology, MATEC Web of Conferences 30, (2015).

8. M. Ovsik, L. Hylova, D. Manas, M. Manas, M. Stanek, MATEC Web of Conferences 76, (2016). 